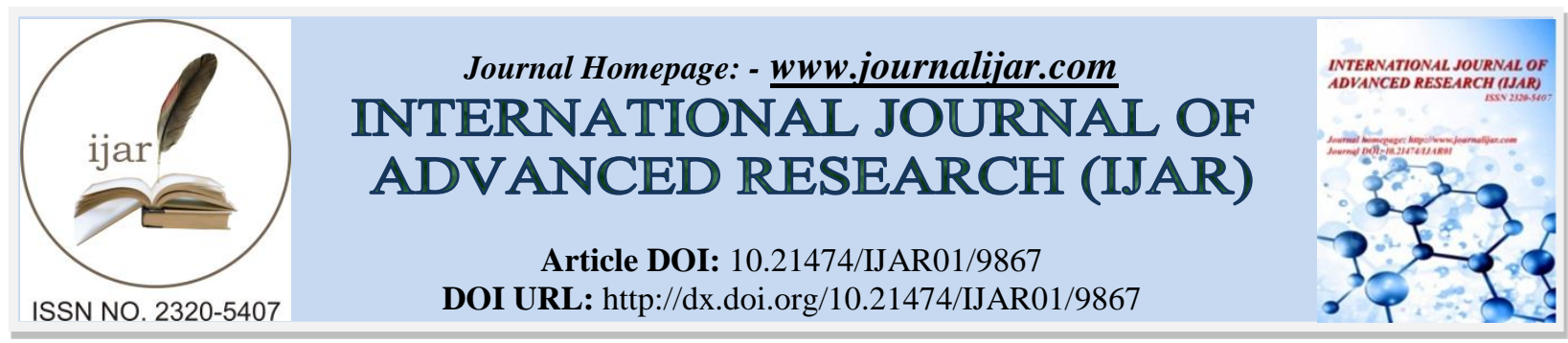

RESEARCH ARTICLE

\title{
A CASE REPORT ON PNEUMOMEDIASTINUM, PNEUMOTHORAX \& SUBCUTANEOUS EMPHYSEMA FOLLOWING DROWNING.
}

\author{
Dr. Sujoy Das Thakur, Dr. Sayani Banerjee, Dr. Santosh Kumar Singh and Dr. Abbas Naqvi.
}

\section{Manuscript Info}

Manuscript History

Received: 08 August 2019

Final Accepted: 10 September 2019

Published: October 2019

Key words:-

Pneumomediastinum, pneumothorax, subcutaneous emphysema, drowning, barotrauma.

\begin{abstract}
We report a case of 18 year old male patient with alleged history of drowning evacuated from water within 2 minutes of submersion, needed initial resuscitation and ventilator support. Chest x-ray and CT chest diagnosed him with pneumomediastinum, bilateral pneumothorax and subcutaneous emphysema. Incidence of pneumomediastinum is a rare. It is usually managed conservatively. We are considering our case to be secondary pneumomediastinum as it developed following drowning and positive pressure ventilation. Though in literature several cases allowed to be reported as spontaneous pneumomediastinum following drowning or other causes, even when a possible causative factor was identified. Good prognostic indicators in case of near drowning in the emergency include GCS $>5 / 15$, short submersion time and spontaneous respiration and cardiac activity. Though our had respiratory failure, he was managed conservatively successfully and discharged home. Presence of pneumomediastinum, pneumothorax are not poor prognostic indicators per se in case of near drowning and can be treated conservatively.
\end{abstract}

Copy Right, IJAR, 2019,. All rights reserved.

\section{Introduction:-}

Pneumomediastinum is abnormal presence of extraluminal gas within the mediastinum. Gas may originate from the lungs/ alveolar space, trachea, central bronchi, esophagus, and peritoneal cavity and track from the mediastinum to the neck or abdomen. In 1819, Laennec first described the term "Pneumomediastinum" (PM) as a consequence of traumatic injury. Spontaneous pneumomediastinum (SPM) was reported in 1939 by Hamman, for whom the Hamman sign is named. It has also been described as mediastinal emphysema ${ }^{1,2,3}$. In the setting of trauma, if pneumomediastinum is visible on chest $\mathrm{X}$-ray it is termed overt pneumomediastinum whereas if it is only visible on CT then it is termed occult pneumomediastinum ${ }^{4}$. Spontaneous pneumomediastinum (SPM) refers to the presence of air into the mediastinum in otherwise healthy subjects without an obvious causative factor such as an operation, air following a viscous perforation, presence of infection or trauma, in absence of mechanical ventilation or valsalva manoeuvre or any explicable cause ${ }^{5,6}$. The presence of air in the mediastinum is considered as secondary pneumomediastinum when a causative factor is identified. Respiratory diseases may lead to pneumomediastinum, especially at periods of exacerbation with excessive coughing i.e., in asthma or respiratory infections ${ }^{7,8,9}$, interstitial lung disease, COPD, bronchiectasis, lung cysts, lung malignancy, excessive vomiting, and trauma (including iatrogenic), recreational drugs, such as cocaine, marijuana, methamphetamine ${ }^{10,11}$, vigorous

Corresponding Author:-Dr. Sujoy Das Thakur. 
valsalva manoeuvre, childbirth, rapid ascent of scuba-divers, presence of foreign bodies in the airway with air trapping, anorexia nervosa, sporting activities and inhalation of toxic fumes.

\section{Case presentation:}

We report a case of 18 year old male patient with alleged history of near drowning, evacuated from water within 2 minutes of submersion, primarily resuscitated, intubated and then brought to us with mechanical ventilator support, in view of deteriorating sensorium and recurrent seizures. On presentation: Patient's GCS was $\mathrm{E}_{4} \mathrm{~V}_{\mathrm{t}} \mathrm{M}_{5}$, Pupils Bilaterally $3 \mathrm{~mm}$ reactive to light, $\mathrm{BP}=120 / 80 \mathrm{mmHg}, \mathrm{HR}=102 / \mathrm{min}, \mathrm{SpO}_{2}=98 \%\left(\mathrm{FiO}_{2}=1.0\right), \mathrm{RR}=20 / \mathrm{min}$, Capillary blood glucose $=82 \mathrm{mg} / \mathrm{dl}$.

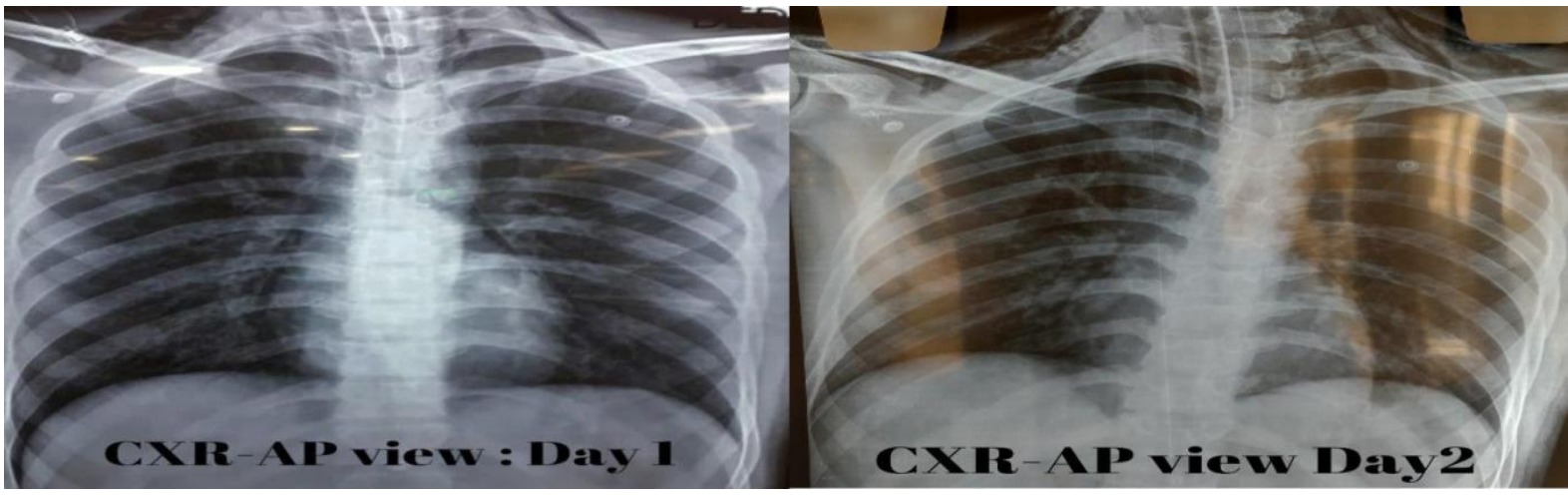

Figure I:-CXR-AP view Da1 vs Day2

Patient was admitted in critical care, managed with continuation of lung protective ventilation, intravenous broad spectrum antibiotic, intravenous antiepileptic (levetiracetam), and other supportive management. CXR-AP view was suggestive of pneumomediastinum and bilateral surgical emphysema. So, HRCT chest was done which reported as: bilateral small pneumothoraxes with pneumomediastinum. Interstitial emphysema in right middle lobe and lingula most likely secondary to pulmonary barotrauma (alveolar rupture). Changes of pulmonary oedema with small consolidations (secondary to aspiration) in both lower lobes. Patient was managed conservatively and in follow up chest radiographs pneumomediastinum resolved.

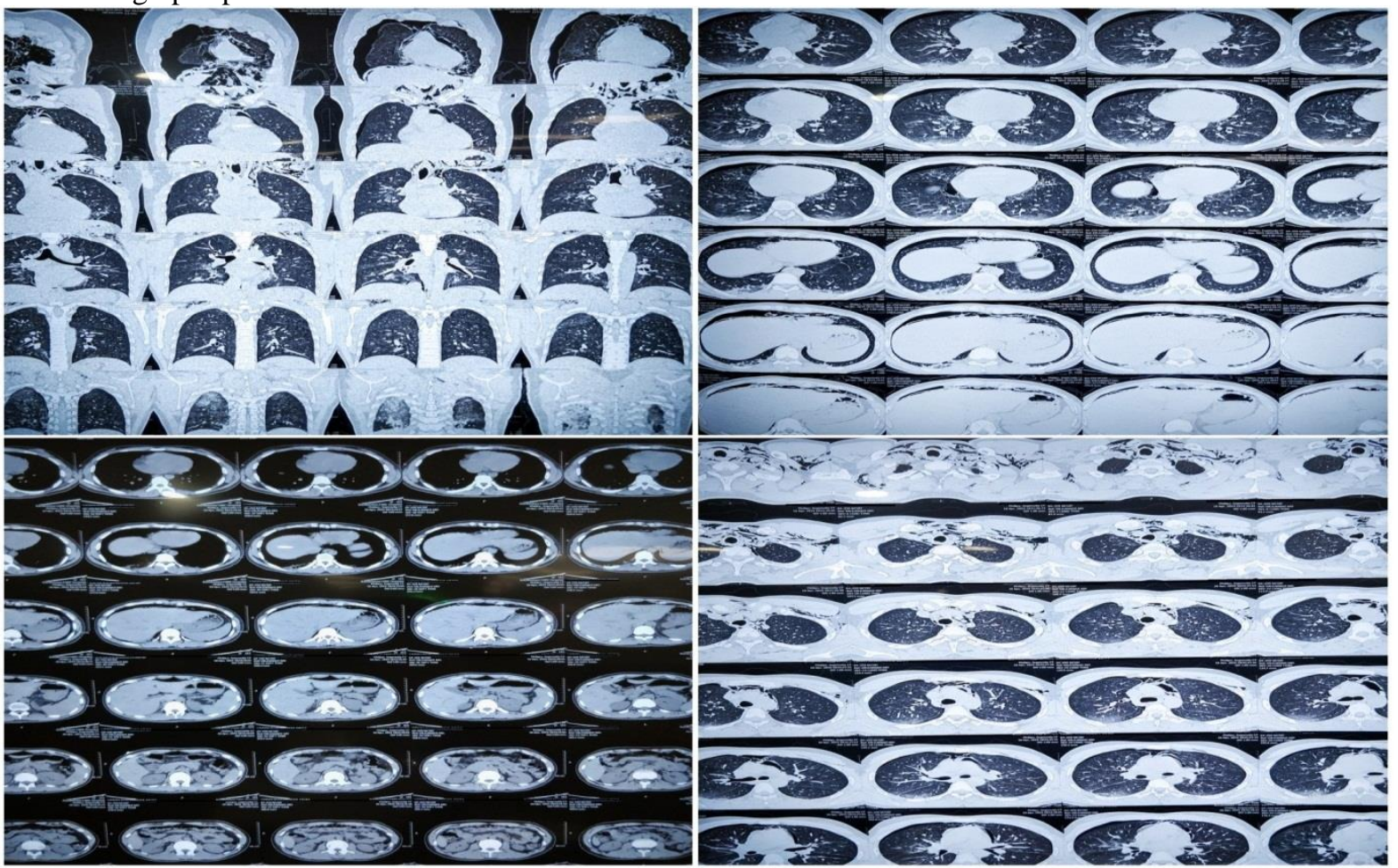

Figure II:-CT Thorax 
Intial total leukocyte count was 16100 /cumm which came down to 7,700/cumm with broad spectrum antibiotics. Kidney function test \& liver function test were within normal limits. Patient Improved with conservative management, extubated on day3 and discharged healthy on day 7.

\section{Discussion:-}

Drowning is the 3rd leading cause of unintentional injury death worldwide, accounting for $7 \%$ of all injury related deaths. There are an estimated 3,60,000 annual drowning deaths worldwide ${ }^{12}$. Global estimates may significantly underestimate the actual public health problem related to drowning. Children, males and individuals with increased access to water are most at risk of drowning. Several of the most densely populated nations in the world fail to report nonfatal drowning incidents. This, along with the fact that in many instances no attempt is made to resuscitate at the scene and that many cases are never brought to medical attention, renders accurate worldwide incidence approximation and classification virtually impossible. The overall incidence of drowning has an estimated range of 20-500 times the rate of fatal drowning.

At the 2002 World Congress on Drowning held in Amsterdam, a group of experts proposed a new consensus definition for drowning i.e. drowning is a process resulting in primary respiratory impairment from submersion in a liquid medium ${ }^{13}$. Implicit in this definition is that a liquid-air interface is present at the entrance to the victim's airway, which prevents the individual from breathing oxygen. All other terms "wet drowning", "dry drowning", "active or passive drowning", "near-drowning", "secondary drowning", and "silent drowning" are only for references to minimize confusion and to allow more accurate analysis and comparison of studies, to draw more meaningful conclusions from pooled data, and improved the ease of surveillance and prevention activities.

As we are discussing our case of pneumomediastinum, i.e. presence of air in mediastinum or mediastinal emphysema and bilateral pneumothorax, i.e. presence of air in pleural cavities following near drowning It must be noted that in literature several reports, the wider term spontaneous pneumomediastinum, has a more favourable outcome and even more rare compared to secondary pneumomediastinum, has been allowed even when a possible causative factor is identified ${ }^{1}$.

INCIDENCE: The pneumomediastinum, spontaneous as well as secondary, is a rare entity, diagnosed in 1/44,500 of accident and emergency attendances or $1 / 100,000$ of natural births, being more frequent in children (1/800$1 / 15,500)^{8}$. Others, report an incidence of $1 / 25,000$ in ages between 5-34 years ${ }^{14}$. The majority of patients are males accounting for $76 \%$ of cases ${ }^{1}$.

\section{Clinical presentation:}

Pneumomediastinum is usually more common in young patients ${ }^{2,6,8}$. One possible explanation is the fact that in young subjects the mediastinal tissues are loose and flaccid, whereas in the elderly group the planes and sheaths are fibrosed, making air migration more difficult to occur. It is also more prevalent in males ( $7 / 10$ cases) $)^{2,8}$ and in patients with preexisting asthma or other respiratory diseases ${ }^{2,5,6}$. A tall, lean, male body habitus is generally considered as the most probable to be presented with pneumomediastinum; however, obese patients are not spared from this pathology.

In Damore's series, the most common symptoms seen with pneumomediastinum (PM) were subcutaneous emphysema $\left(76 \%\right.$ of patients) and neck or chest pain $\left(38 \%\right.$ of patients) ${ }^{15}$. In a narrative literature review of 216 patients, Gasser et al (2017) note that chest pain (55\%), neck pain or sore throat (53\%) and dyspnea (41\%) were the most frequently identified symptoms ${ }^{16}$. Other presenting signs include rhinolalia (nasally sounding voice, which occurs because of dissection of air into the soft palate), hoarseness and neck swelling, tachycardia, tachypnea or anxiety, presence of Hamman's sign; it is the presence of mediastinal crunch or click present on auscultation over the cardiac apex and the left sternal border synchronous with the heart beat ${ }^{2}$.

Malignant pneumomediastinum is considered the accumulation of a significant amount of air in the mediastinum, causing vessel or tracheal obstruction and inducing respective symptoms and signs of tamponade and decreased venous return. Only few cases of this adverse appearance have been described and on this occurrence may be considered rare. 


\section{Pathophysiology:}

The increase of alveolar pressure, in case of near drowning possibly secondary to initial breath holding immediately following drowning to prevent water to enter the airway, causes them to rupture, therefore releasing air which in turn migrates through the peribronchial and perivascular sheaths to the mediastinum ${ }^{17}$. Another possible explanation for pneumomediastinum is, the abnormal increase of pressure in the mediastinum causing air to dissect in between the mediastinal structures, which support the mediastinal organs. A dramatic decrease in intravascular pressure also can create a relative pressure gradient in the perivascular spaces ${ }^{1}$. The air may then dissect to the neck, upper abdomen or the skin via the loose alveolar fat tissue (subcutaneous emphysema) ${ }^{8}$. Air can also pass the pleura resulting in pneumothorax or the peritoneum resulting in pneumoperitoneum ${ }^{18}$.

\section{Diagnosis:}

Dianosis can be established by a plain anterio-posterior chest $\mathrm{x}$-ray in $90 \%$ cases and rarely a lateral chest $\mathrm{x}$-ray is needed $^{7,10,11}$.CT Chest is used in suspicious cases with pneumomediastinum with inconclusive $\mathrm{x}$-ray chest to confirm the diagnosis, to assess the extent of the pneumomediastinum, (i.e. mild, moderate or severe), to differentiate between pneumomediastinum and pneumopericardium and to diagnose the presence of a pneumothorax missed on a plain chest X-ray in cases with severe subcutaneous emphysema. Ultrasound of the mediastinum is lately applied in the emergency department, to identify pneumomediastinum in cases of high urgency. Based on chest X-ray and CT chest findings many patterns have been identified: thymic sail (elevation of thymus due to air, mainly seen in pediatric population), ring sign (caused by air surrounding the pulmonary artery or either of its main branches), double bronchial wall, continuous diaphragm sign, or air adjacent to hemidiaphragm or spine $e^{1,19,20,21}$.

\section{Management:}

Pneumomediastinum is generally considered a benign entity with good prognosis. Most cases of pneumomediastinum are managed conservatively. After excluding significant underlying pathology, the pneumomediastinum treatment is directed towards symptom relief ${ }^{1,22}$. Analgesics to control pain, anxiolytics, antitussives are commonly used. Administration of $\mathrm{O} 2$ may increase gas absorption by six fold and should therefore be considered. Any underlying exacerbation of a pre-existing pathology such as asthma or COPD should be adequately attended. Asymptomatic patients can be discharged after at least 24 hours of observation. Recurrences have been reported and therefore follow up is advisable.

\section{Conclusion:-}

In coclusion, the case we reported is a rare entity secondary pneumomediatinum, with bilateral pneumothorax and subcutaneous emphysema following near drowning in an young 18 years old male without any pre-existing illness. Good prognostic indicators in case of near drowning in the emergency include GCS $>5 / 15$, short submersion time and spontaneous respiration and cardiac activity ${ }^{23}$. Though our patient was initially needed ventilator support secondary to respiratory failure, he was managed conservatively successfully and discharged home. Presence of pneumomediastinum, pneumothorax are not poor prognostic indicators per se in case of near drowning and can be treated conservatively.

\section{Reference:-}

1. Agut A, Talavera J, Buendia A, et al. Imaging diagnosis - spontaneous pneumomediastinum secondary to primary pulmonary pathology in a dalmatian dog. Vet Radiol Ultrasound 2014.

2. Kobashi Y, Okimoto N, Matsushima T, et al. Comparative study of mediastinal emphysema as determined by etiology. Intern Med 2002;41:277-82.

3. Sahni S, Verma S, Grullon J, et al. Spontaneous pneumomediastinum: time for consensus. N Am J Med Sci 2013;5:460-4.

4. Rezende-Neto JB, Hoffmann J, Al Mahroos M et-al. Occult pneumomediastinum in blunt chest trauma: clinical significance. Injury. 2010;41 (1): 40-3. doi:10.1016/j.injury.2009.06.161.

5. Chiu CY, Wong KS, Yao TC, et al. Asthmatic versus nonasthmatic spontaneous pneumomediastinum in children. Asian Pac J Allergy Immunol 2005;23:19-22.

6. Macia I, Moya J, Ramos R, et al. Spontaneous pneumomediastinum: 41 cases. Eur J Cardiothorac Surg 2007;31:1110-4.

7. Caceres M, Ali SZ, Braud R, et al. Spontaneous pneumomediastinum: a comparative study and review of the literature. Ann Thorac Surg 2008;86:962-6. 
8. Russo A, Del Vecchio C, Zaottini A, et al. Role of emergency thoracic ultrasonography in spontaneous pneumomediastinum. Two case report. G Chir 2012;33:285-96.

9. Chu CM, Leung YY, Hui JY, et al. Spontaneous pneumomediastinum in patients with severe acute respiratory syndrome. Eur Respir J 2004;23:802-4.

10. Perna V, Vilà E, Guelbenzu JJ, et al. Pneumomediastinum: is this really a benign entity? When it can be considered as spontaneous? Our experience in 47 adult patients. Eur J. Cardiothorac Surg 2010;37:573-5.

11. Iyer VN, Joshi AY, Ryu JH. Spontaneous pneumomediastinum: analysis of 62 consecutive adult patients. Mayo Clin Proc 2009;84:417-21.

12. World Health Organization. World Health Organization Global Report on Drowning. Available at http://www.who.int/violence_injury_prevention/global_report_drowning/final_report_full_web.pdf. $\quad 2014$; Accessed: July 1, 2016.

13. Van Beeck EF, Branche CM, Szpilman D, Modell JH, Bierens JJ. A new definition of drowning: towards documentation and prevention of a global public health problem. Bull World Health Organ. 2005 Nov. 83(11):853-6.

14. Jougon JB, Ballester M, Delcambre F, et al. Assessment of spontaneous pneumomediastinum: experience with 12 patients. Ann Thorac Surg 2003;75:1711-4.

15. Damore DT, Dayan PS. Medical causes of pneumomediastinum in children. Clin Pediatr (Phila). 2001 Feb. 40(2):87-91.

16. Gasser CR, Pellaton R, Rochat CP. Pediatric Spontaneous Pneumomediastinum: Narrative Literature Review. Pediatr Emerg Care. 2017 May. 33 (5):370-374.

17. Macklin MT, Macklin CC. Malignant interstitial emphysema of the lungs and mediastinum as an important occult complication in many respiratory diseases and other conditions: an interpretation of the clinical literature in the light of laboratory experiment. Medicine 1944;23:281-358.

18. Pooyan P, Puruckherr M, Summers JA, et al. Pneumomediastinum, pneumopericardium, and epidural pneumatosis in DKA. J Diabetes Complications 2004;18:242-7.

19. Kaneki T, Kubo K, Kawashima A, et al. Spontaneous pneumomediastinum in 33 patients: yield of chest computed tomography for the diagnosis of the mild type. Respiration 2000;67:408-11.

20. Moseley JE. Loculated pneumomediastinum in the newborn. A thymic "spinnaker sail" sign. Radiology 1960;75:788-90.

21. Hammond DI. The "ring-around-the-artery" sign in pneumomediastinum. J Can Assoc Radiol 1984;35:88-9.

22. Koullias GJ, Korkolis DP, Wang XJ, et al. Current assessment and management of spontaneous pneumomediastinum: experience in 24 adult patients. Eur J Cardiothorac Surg 2004;25:852-5.

23. Meacher S. Managing near drowning of adults within the emergency department. Austral Emerg Nurs $\mathbf{J}$ 2006;9:3-9. 